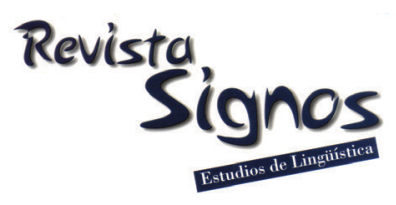

\title{
Estrategias argumentativas en niños pequeños: Un estudio a partir de las disputas durante el juego en contextos escolares
}

\author{
Argumentative strategies in young children: \\ An study of disputes during play in school contexts
}

\author{
Maia Julieta \\ Migdalek \\ CONICET \\ Universidad de Buenos Aires \\ Argentina \\ maiamig@hotmail.com
}

\author{
Cristián \\ Santibáñez Yáñez \\ Universidad Diego Portales \\ CHILE \\ cristian.santibanez@udp.cl
}

Celia Renata

Rosemberg

CONICET

Universidad de Buenos Aires

ArgentinA

crrosem@hotmail.com

Recibido: 25-I-2013 / Aceptado: 14-X-2013

\section{Resumen}

En este trabajo se pone a prueba un sistema de categorías para analizar las estrategias argumentativas que despliegan niños en situaciones de juego en el contexto educativo. Se indaga si el sistema resulta adecuado para describir y evaluar las estrategias argumentativas de niños de 3, 4 y 5 años en el contexto del jardín de infantes. El estudio empírico se desarrolla a partir de la observación de interacciones de niños en situaciones naturales de juego en jardines de infantes de la Ciudad de Buenos Aires, Argentina. El estudio ha permitido concluir la validez del sistema para contextos institucionales. Asimismo, el análisis muestra que los niños producen mayormente expresiones de oposición argumentativa sin desplegar argumentos estructurados (razón-conclusión), lo que podría ser característico de una fase protoargumentativa, y que entre los niños de 5 años se observa un uso más recurrente del esquema argumentativo de apelación a la mayoría.

Palabras Clave: Argumentación, juego, niños, oposición, puntos de vista. 


\begin{abstract}
In this paper, a model is tested for its adequacy to describe and evaluate argumentative strategies in 3, 4 and 5-year old children in a kindergarten context. The data consist of the observation of children's interactions in natural play settings in kindergartens in Buenos Aires city. The results show that the proposed model is valid for institutional contexts. Furthermore, the analysis reveals that children mainly produce statements of argumentative opposition without using structured arguments (reason-conclusion), which could be a characteristic of a proto-argumentative phase. It also shows that among 5-year olds the ad populum argumentative scheme is used recurrently.
\end{abstract}

Key Words: Key Words: Argumentation, children, opposition, play, points of view.

\title{
INTRODUCCIÓN
}

No es un misterio que la teoría de la argumentación ha sido elaborada a partir de evidencia proveniente de producción argumentativa de hablantes adultos (Tindale, 1999, 2004; Johnson, 2000; van Eemeren \& Grootendors, 2004; van Eemeren, 2010; Walton, Reed \& Macgano, 2008). Sin embargo, poco a poco se instala el interés práctico y teórico por conocer cómo emerge y se desarrolla esta competencia. Este conocimiento permitiría una comprensión más adecuada de lo que significa argumentar.

Con el objeto de estudiar la competencia y práctica argumentativa en infantes, se ha elaborado un sistema de categorías para analizar las habilidades argumentativas de niños preescolares desde una perspectiva que integra las teorías socioculturales del desarrollo humano (Vygotski, 1964; Luria, 1995; Wertsch, 1998), los modelos psicolingüísticos actuales con base sociocultural (Nelson, 1996; Tomasello, 2008, 2010) y la psicología del razonamiento (Clément, 2010; Evans, 2010; Mercier, 2011; Mercier \& Sperber, 2011; Stanovich, 2011). Dicho sistema fue generado inductivamente a partir del análisis de disputas ocurridas en situaciones naturales de juego de las que participaron, en sus hogares, niños de 4 años de población urbano marginada y de sectores medios de la Ciudad de Buenos Aires, Argentina (Migdalek \& Arrúe, 2012a). El sistema conceptualiza las intervenciones infantiles que exponen puntos de vista explícitos o implícitos-, y las que presentan un comportamiento general de oposición conversacional junto con algún tipo de estrategia argumentativa.

En línea con los postulados de la psicología sociocultural (Wertsch, 1998; Cole, 1999; Del Río \& Álvarez, 2001) y de la Sociolingüística Interaccional (Gumperz, 1982 , 1984) relativos a la estrecha interdependencia entre el lenguaje, la cognición y la actividad contextuada, resulta pertinente analizar si el sistema de categorías elaborado puede también dar cuenta de las argumentaciones en contextos educativos institucionales. Con el objeto de contribuir con evidencia empírica de diversos contextos a la elaboración de una teoría acerca del desarrollo de la competencia 
argumentativa, el presente artículo se propone: a) detectar, describir y analizar las estrategias argumentativas empleadas por niños pequeños en disputas entre pares en el contexto del jardín de infantes; b) evaluar la validez del sistema de categorías correspondiente a las estrategias argumentativas empleadas por niños de 4 años de diversos grupos socioeconómicos en disputas durante situaciones de juego en el contexto del hogar (Migdalek \& Arrúe, 2012a) para estudiar la argumentación infantil en situaciones de juego en el marco institucional del jardines de infantes a los que asisten niños de poblaciones urbano marginadas; c) analizar eventuales diferencias en las estrategias argumentativas utilizadas en niños y niñas de 3, 4 y 5 años de edad respectivamente, en disputas entre pares en contextos de educación pre-escolar; d) observar y analizar el uso implícito o explícito de la noción de punto de vista en situaciones controversiales en el contexto de juegos de niños.

\section{Marco teórico general}

La interacción que se produce en las actividades situadas constituye el espacio privilegiado para la adquisición y desarrollo del lenguaje así como de sus subcompetencias, como es argumentar. Numerosas investigaciones coinciden en que en el encuentro social es donde se construyen, modifican y afianzan las competencias lingüísticas, a través de los procesos de intercomprensión, negociación del sentido y posicionamientos recíprocos (Bruner, 1986; Mondada \& Pekarek, 2001; Pekarek, 2006). El juego, actividad rectora y social de los primeros años de la vida de los niños (Leontiev, 1981; Tomasello, 2008), constituye un marco en el que estas dinámicas son posibles de observar.

En las situaciones de juego, el lenguaje es uno de los medios centrales para crear y sostener en la interacción con otros las reglas del juego y desplegar la secuencia narrativa (Seidman, Nelson \& Gruendel, 1986; Nelson, 1996); esta situación de interacción requiere que el niño use sus recursos lingüísticos para comunicarse con otros de modo tal que éstos puedan comprender las intenciones que lo motivan (Rosemberg, 2008). Se ha señalado que el juego dramático es un contexto propicio para la argumentación dado que las intenciones de los jugadores, el plan de juego y las acciones que lo conforman, son negociados y co-construidos (Zadunaisky Ehrlich \& Blum-Kulka, 2010).

Los trabajos acerca del desarrollo de las competencias discusivas concuerdan en que la adquisición de la argumentación, como forma elaborada, se produce en la adolescencia (Silvestri, 2001). Sin embargo, ya a la edad de 2 años es posible observar enunciados con la función de argumentar. Recientes estudios (Bernard, Mercier \& Clément, 2012) muestran que los niños a partir de los 3 años ya son sensibles, en su rol de audiencia, al conectivo 'porque' como desencadenante de argumentación. Esta producción y comprensión argumentativa en niños pequeños ha sido denominada 'protoargumentación', en tanto se carece de ciertas operaciones 
consideradas específicas del argumentar (Eisenberg, 1987; Peronard, 1991; Crespo, 1995; Silvestri, 2001). En la 'protoargumentación' se observan enunciados que buscan convencer al interlocutor de la verdad de aquello que se afirma o niega, justificar una acción o un pedido, sin la estructura completa razón-conclusión (van Dijk, 1983; Eisenberg, 1987; Peronard, 1991). En el transcurso de la niñez, el niño va ampliando sus habilidades argumentativas, siendo necesario para el completo desarrollo de la competencia argumentativa que su entorno directo e indirecto lo propicie (Silvestri, 2001; Faigenbaum, 2012). En este sentido, resulta relevante estudiar la argumentación en el contexto del jardín de infantes. Conocer el modo en que los niños sostienen sus puntos de vista en situaciones naturales de interacción puede posibilitar el desarrollo posterior de diseños didácticos orientados al fortalecimiento de la subcompetencia argumentativa.

Investigaciones sobre el desarrollo cognitivo de los niños evidenciaron que ya a los 3 años pueden comprender la especificidad contextual de las reglas normativas en el juego dramático y apelar a ellas para sancionar comportamientos inadecuados de los personajes en el marco ficcional (Wyman, Rakoczy \& Tomasello, 2009). Otros estudios mostraron que bebés de 13 meses, e incluso de 7 meses, poseen una incipiente capacidad metarrepresentacional que les permite atribuir creencias a los agentes (Surian, Caldi \& Sperber, 2007; Kovács, Téglás \& Endres, 2010), habilidad implicada en la comprensión y producción de argumentaciones (Desalles, 2007).

Diversas investigaciones han analizado la argumentación infantil. Dunn y Munn (1987) estudiaron las justificaciones que niños ingleses de entre 18 meses a 3 años proveen en disputas con hermanos mayores y madres en sus hogares. Los niños inicialmente sostienen sus puntos de vista a través de una estructura circular, con justificaciones basadas en sus sentimientos, para luego comenzar a apelar a reglas sociales y a consecuencias materiales para apoyar sus posiciones. Por su parte, Eisenberg (1987) en su investigación longitudinal con dos niñas estadounidenses hablantes de español, desde los 22 meses hasta los 3 años, identificó ciertas estrategias persuasivas, tales como la insistencia, el soporte verbal, la mitigación, la apelación a otro individuo, la amenaza, el abuso verbal y la oferta de un compromiso. Asimismo, mostró que el uso de estas estrategias varía según el rol funcional que los niños toman en la disputa -proponente u oponente- y la identidad de participantes - pares o padres-.

Entre la amplia producción de Stein y colaboradores cabe mencionar el trabajo de Stein y Albro (2001) donde estudiaron la argumentación de niños preescolares estadounidenses a partir de conflictos con sus padres. Señalan que dichos conflictos constituyen el marco para el desarrollo de las habilidades argumentativas y enfatizan la importancia de los objetivos personales y sociales, y las emociones en el curso de la argumentación.

También Cobb-Moore, Danby y Farell (2009) estudiaron la regulación verbal de situaciones de juego entre pares en un Jardín de Infantes en Australia. El análisis reveló 
que los niños se valen de cuatro prácticas interaccionales como formas de regulación: 1) el reclamo de la posesión de objetos y lugares de juego; 2) el desarrollo o uso de reglas preexistentes y del orden social para controlar la interacción con sus pares; 3 ) el uso estratégico del lenguaje para regular las acciones de aquellos que están a su alrededor; 4) la creación y utilización de categorías de membresía para incluir o excluir a otros y también para controlar y participar en la interacción en desarrollo. Por otra parte, Zadunaisky Ehrlich y Blum-Kulka (2010) analizaron eventos argumentativos en interacciones naturales entre niños preescolares de 3 centros educativos en Israel. Los resultados muestran que los niños gestionan el evento argumentativo mediante estrategias efectivas y de base racional propias de la cultura adulta, al tiempo que negocian sus relaciones sociales.

En el ámbito latinoamericano, Peronard (1991) investigó los precursores del discurso argumentativo en cinco niños chilenos, durante los primeros 4 años de su vida. Los resultados mostraron que los argumentos se construyen, en el inicio, en la secuencia dialógica y son desencadenados por los padres. Paulatinamente los niños van cobrando autonomía en su producción, siendo el orden de los mismos conclusiónrazón. En cuanto al contenido de los argumentos, Peronard (1991) señala que mayormente se trata de 'argumentos de acción' y los categoriza en relativos al mundo material, al mundo cultural o al mundo psicológico. En una investigación también realizada en Chile, Crespo (1995) exploró la adquisición espontánea del argumento en un niño desde los 2 hasta los 3 años de edad y su posterior enriquecimiento en otros dos niños desde los 5 hasta los 6 años de edad, utilizando la clasificación de 'tipos de argumentos' de Perelman y Olbrechts Tyteca (1989). Sus resultados concuerdan con los de Peronard (1991), en tanto los primeros argumentos son predominantemente argumentos de acción. Asimismo, señala que lo más frecuente es que estén basados en la causalidad, luego en el modelo, la ilustración y finalmente, la coexistencia.

\section{Metodología}

\subsection{La estrategia general}

Las situaciones analizadas en este trabajo son parte de una investigación más amplia de carácter cualitativo acerca de la regulación de las situaciones de juego en el jardín de infantes ${ }^{1}$. Participaron 54 niños de 3 años distribuidos en dos salas, 63 niños de 4 años que asistían a otras dos salas y 122 niños de 5 años de edad distribuidos en 4 salas distintas. Las maestras a cargo de cada sala informaron que ninguno de los niños presentaba retrasos madurativos.

La unidad de análisis está conformada por situaciones de juego en rincones observadas en las escuelas infantiles ${ }^{2}$, a las que asisten niños de poblaciones urbanomarginadas de la Ciudad de Buenos Aires y el Conurbano Bonaerense, Argentina ${ }^{3}$. La elección de dicha actividad reside en su carácter distintivo en el jardín de infantes en tanto en función de ella se configura el espacio en las aulas de este nivel educativo. 
Dentro de las situaciones de juego, se consideraron, como unidad de análisis menor, las disputas que se producían entre los niños en el juego. Las definimos como el encuentro de dos puntos de vista opuestos. Para determinar su alcance se consideró su resolución tanto a través de un acuerdo verbal como de un curso de acción posterior a favor de uno de los puntos de vista enfrentados.

En el presente trabajo se analizaron 15 situaciones de juego en rincones -5 por cada edad considerada- durante las que se registraron 124 disputas entre pares. El total se distribuyó de la siguiente manera: 32 disputas entre niños de 3 años; 29 disputas entre niños de 4 años y 63 entre pequeños de 5 años.

\subsection{Procedimientos de obtención de información}

Los datos fueron obtenidos mediante la observación no participante en las situaciones de juego y registrados en videofilmaciones. Las investigadoras que realizaron la toma de datos eran familiares para las comunidades educativas de los jardines ya que dichas instituciones participaban de un programa de intervención para el desarrollo lingǘstico y cognitivo de los niños llevado a cabo por el mismo equipo de investigación. En este sentido, para cada sala había una investigadoraorientadora asignada que trabajaba en la capacitación en servicio de la docente a cargo del grupo y asistía frecuentemente a observar actividades del programa de desarrollo lingüístico y cognitivo. Los distintos grupos de niños estaban habituados la presencia de la investigadora-orientadora en las aulas, quien filmaba o audiogrababa diversas actividades. El registro de las situaciones de juego en rincones se realizó entre julio y noviembre de un año lectivo. Se estipuló que las orientadoras-investigadoras no interviniesen generando situaciones lúdicas. Sin embargo, sí podían responder ante una eventual demanda de los niños, porque se consideró que no responder a las demandas infantiles podía afectar la ecología de la situación. Asimismo, dado que se trataba de situaciones naturales de juego en las que había un alto nivel de ruido ambiente, se incluyó un grabador para intentar garantizar la calidad sonora de la muestra.

Para la transcripción de la información empírica se han seguido las pautas propuestas por el Análisis de la Conversación (Tusón, 1995) consignadas en el anexo, pues permiten dar cuenta en la interacción de los componentes verbales y no verbales (los gestos), la dirección de la mirada, el posicionamiento corporal, la entonación. Estos últimos constituyen 'pistas de contextualización' en tanto guían la negociación del significado en la interacción (Gumperz, 1982, 1984).

\subsection{Procedimientos de análisis}

En esta investigación se empleó de modo heurístico el sistema de categorías de estrategias argumentativas infantiles elaborado por Migdalek y Arrúe (2012a). Dichas categorías fueron desarrolladas de modo inductivo a través del procedimiento cualitativo sistemático de comparación propuesto por la Grounded Theory (Glaser 
\& Strauss, 1967; Strauss \& Corbin, 1991) a partir de un corpus de 480 horas de observación de situaciones de la vida cotidiana de niños de 4 años de distintos estratos socioculturales y económicos de la Ciudad de Buenos Aires, Argentina. Las estrategias argumentativas que comprende dicho sistema son la reiteración del punto de vista sostenido; la anticipación de cursos de acción; la descripción de objetos, situaciones o estados internos; la narración; la apelación a la autoridad; la generalización; la mostración; la propuesta alternativa; la mitigación y la agresión verbal.

El procedimiento de análisis de intercambios en el juego involucró los siguientes pasos:

a) Lectura de las situaciones de juego en las salas de niños de 4 años.

b) Identificación de disputas en el marco de dichas interacciones. Se consideraron todos los intercambios en los que se identificaron puntos de vista enfrentados.

c) Clasificación de las estrategias verbales utilizadas por los niños para defender su punto de vista a partir del sistema de categorías previo (Migdalek \& Arrúe, 2012a). Siguiendo el procedimiento de comparación constante (Strauss \& Corbin, 1991), se compararon las estrategias argumentativas identificadas en las disputas en el contexto del hogar con las identificadas en el contexto del jardín de infantes. Así, por ejemplo, se pudo establecer la recurrencia de la categoría de Anticipación, hallada en el contexto del hogar, en el contexto del jardín, en tanto los incidentes contemplados comparten sus características esenciales, de orden semántico o pragmático. A continuación ilustramos el procedimiento de análisis.

Estrategia de Anticipación correspondiente al corpus de juego en el contexto del hogar (Migdalek \& Arrúe, 2012a). Ejemplo (1) Agustín, 4 años, población urbana marginada, juega con un amigo, Carlitos, de 4 años, a la pelota.

Carlitos: ¡Dale, Agus, juguemos ahora!

Agustín: Con esta no, con esta no [se refiere a la pelota grande de cuero] si no me va a doler la cabeza, la panza. Despacito golpea porque si no me vas

a...

Estrategia de Anticipación correspondiente al corpus de juego en el contexto del jardín de infantes. Ejemplo (2) Diego y Armando construyen 'la casa de los marcianos' con bloques de madera.

Diego: así mira\ [Acercándose para agarrar la madera.]

Armando: no porque si pongo así se cae esto y esto [Señala con el bloque dos partes de la construcción.] 
En el ejemplo (1) se observa que Agustín proyecta las consecuencias negativas -esquema argumentativo de apelación a las consecuencias negativas (Walton, 2006)que puede tener el uso de la pelota de cuero para fundamentar, primero, el pedido de cambiar de pelota y luego, el pedido de que su amigo patee despacio. Lo mismo se observa en el ejemplo (2), en tanto Armando proyecta la caída de la construcción como consecuencia negativa de proceder como sugiere su compañero de juego. Asimismo, los incidentes comparten una estructuración sintáctica similar: en ambos se emplea una construcción condicional encabezada por la forma 'si'.

De este modo, la metodología empleada permite realizar una operación de abstracción de las características particulares de los casos estudiados para incluirlos en una categoría común si se identifica como recurrente el rasgo esencial de la categoría, que las distingue de las demás. Así, el ejemplo (3), que se presenta a continuación también corresponde a la categoría de Anticipación pues, si bien difiere en cuanto a su contenido léxico y a su realización sintáctica, comparte la característica común de implicar la proyección de las consecuencias de un curso de acción determinado.

Estrategia de Anticipación correspondiente al corpus de juego en el contexto del jardín de infantes. Ejemplo (3) Brenda y Aron juegan en el rincón de dramatizaciones.

(3)

Brenda: [Toma un cacharro que estaba sobre la hornalla.] ACÁ HAY OTRA GELATINA/

\section{Aron: [A Melany] te va a quemar vo $\backslash$. te va a queMAAA/}

d) Una vez categorizadas todas las estrategias argumentativas presentes en las disputas registradas en el contexto del jardín de infantes, se observó que en el nuevo corpus de datos de disputas en el jardín de infantes se hallaban representadas todas las categorías de estrategias comprendidas en el sistema de estrategias argumentativas en el contexto del hogar (Migdalek \& Arrúe, 2012a).

Se siguió el mismo procedimiento para el análisis de las disputas registradas en las salas de niños de 3 años y de 5 años.

El análisis que dio lugar a la categorización de las estrategias que los niños empleaban para sostener sus puntos de vista fue realizado conjuntamente por dos de los autores del trabajo. Cada uno de ellos codificó independientemente el 25\% de los casos. En un 90,47\% de los casos acordaron en los códigos asignados. Luego de discutir y acordar criterios para categorizar los casos periféricos, el $50 \%$ restante de las situaciones fue codificado por la primera autora del trabajo.

\section{Resultados}

El análisis inductivo de los datos realizado por medio de un procedimiento cualitativo de comparación constante (Glaser \& Strauss, 1967; Strauss \& Corbin, 1991) 
evidencia que el sistema de categorías desarrollado en el contexto del hogar (Migdalek \& Arrúe, 2012a) resulta una herramienta pertinente para dar cuenta de las estrategias argumentativas en contextos institucionales. Solo una categoría, la agresión verbal, no fue registrada en los intercambios. Los resultados muestran la forma particular en que dichas estrategias se despliegan en la interacción en las disputas en este contexto. Asimismo, se observa que las mismas categorías pueden emplearse para analizar disputas entre niños de 3 a 5 años, considerando diferencias en la elaboración y usos de las estrategias en los tres grupos etarios.

Asimismo, el análisis cualitativo por comparación constante (Glaser \& Strauss, 1967; Strauss \& Corbin, 1991) de las disputas en los niños de 3, 4 y 5 años muestra que mientras en algunas situaciones ellos solo expresan puntos de vista enfrentados, en otras despliegan, además, algún tipo de estrategia argumentativa que les permite sostenerlos. Se presenta a continuación el análisis de situaciones de disputa en las que los niños solo expresan posiciones opuestas; luego, en el apartado 4.2 se analizan e ilustran las diversas estrategias argumentativas a las que recurren los niños para sostener sus posiciones.

\subsection{Expresión de posiciones opuestas sin estrategias argumentativas}

Los ejemplos siguientes corresponden a situaciones de juego en las que los niños discuten sin presentar argumentos para apoyar su punto de vista. Entendemos como puntos de vista opuestos la manifestación verbal de rechazo a una acción o enunciado previos. El primero fue registrado en una sala de niños de 3 años. Los niños se han distribuido en pequeños grupos alrededor de mesas en las que hay distintos juguetes réplica correspondientess al guión del 'doctor'. Un subgrupo compuesto por dos niñas, Rocío y Antonella, y un niño, Pedro, se encontraba curando a un bebote. Ingrid que se encontraba jugando en otra mesa, se acerca a ellos.

(4)

Ingrid: [Busca algo primero en la estantería y luego intenta sacarle algo a Pedro.]

Pedro: e: NO: [Ingrid se aleja, deambula brevemente. Luego le quita algo a Rocío y Pedro.]

\section{Rocío: dame E:so\}

Pedro: [a Ingrid] NO::

Pedro y Rocío: [a coro] NO::: [Ingrid se aleja con el objeto que les sacó a los niños, gira para mirarlos y se dirige a otro espacio de juego.] 
Como se observa en el ejemplo (4), Rocío y Pedro rechazan las acciones de Ingrid a través de la mera expresión de la forma negativa 'no'. Asimismo, Rocío reclama un objeto para sí sin fundamento alguno.

Entre los niños de 4 años también se registró este comportamiento de oposición. El ejemplo que lo ilustra fue registrado durante un juego de mesa del que participaban dos niños, Martín y Carlos. El juego consiste en pescar con una espátula de cocinero fichas de diversos tipos de peces que se encuentran dentro de un tablero con forma de ballena. Carlos acusa a Martín de estar haciendo trampa y éste rechaza la acusación solo mediante el uso de la forma negativa.

(5)

Carlos: no\vo: .. bueno (nos equivocamos) [cambia los platos]

Martín: ya está? [Carlos junta peces] EH::: \[risas]

Carlos: yo no estoy haciendo trampa \VO estás haciendo trampa

Martín: NO::

Carlos: mucha trampal

Ahora bien, en cuanto al nivel de la formulación, se observan similitudes y diferencias en la expresión del punto de vista en los ejemplos presentados. Por una parte, en los dos casos se observa el punto de vista de modo explícito -Rocío: dame E:so; Martín: NO:: $\backslash$; Diego: NO:: - Asimismo, se registran en ellos el alargamiento vocálico y la entonación enfática, que constituyen modos no verbales de afirmar el punto de vista. Por otra parte, la formulación observada en los niños de 4 años muestra una mayor elaboración, en tanto remite a las reglas del juego -propias del espacio institucional o del formato (Bruner, 1986) del mismo-, mientras que en los casos correspondientes a los niños de 3 años se encuentra ligada al contexto inmediato de actividad.

\subsection{Sostenimiento del punto de vista mediante estrategias argumentativas}

En muchas de las disputas ocurridas durante el juego, los niños despliegan diversas estrategias argumentativas para sostener y persuadir a sus compañeros de juego de llevar a cabo determinadas acciones y compartir sus posiciones. Las estrategias identificadas coinciden con las identificadas por Migdalek y Arrúe (2012a) en disputas entre niños registradas en situaciones de juego en el contexto del hogar. Presentamos a continuación los intercambios que ilustran las categorías identificadas.

\subsubsection{Reiteración}

En los siguientes ejemplos se observa la repetición de elementos léxicos o 
construcciones sintácticas como estrategia principal para sostener el punto de vista. El ejemplo (6) corresponde a niños de 3 años. Fue registrado durante una situación de juego dramático en la que los niños se encontraban representado el guión del doctor con diversos juguetes réplica. Ingrid, se encontraba sola en una mesa atendiendo a un bebote, cuando Pablo se acercó a su mesa y le puso una inyección al bebote de Ingrid.

(6)

Pablo: [Se acerca a la muñeca de Ingrid y le pone una inyección con una jeringa]

Ingrid: no no no [Ingrid empuja suavemente la mano con la jeringa. Pablo se va.]

Como puede apreciarse en el fragmento anterior, Ingrid rechaza la acción de Pablo mediante la repetición de la forma 'no'.

Presentamos un caso correspondiente a esta estrategia argumentativa en una disputa (ejemplo 7) ocurrida entre niños de 5 años que se encontraban jugando en el rincón de construcciones. Tres niños, Lucas, Armando y Diego, se encontraban construyendo 'la casa de los marcianos'. Alejandro, que también se encontraba en el rincón pero no construía activamente, propone asignarles una función a las piezas que sus compañeros han añadido a la construcción.

Alejandro: ES EL MOTOR ES EL MOTOR\. ése es el motor que tenía una espada [Señala] =es el motor que tenía una espada $\backslash=$

Armando.: $=\mathrm{NO} /::=[$ Niega con la cabeza $] ..$ alejo no juegal

En el intercambio anterior es posible observar cómo Alejandro reitera la función que quiere asignarles a las piezas para persuadir a sus compañeros de que la acepten. Sin embargo, Armando, líder del grupo, rechaza esta designación y lo excluye del juego.

Ahora bien, de los fragmentos anteriores es posible notar que el punto de vista sostenido por los niños puede estar explícito, como en los ejemplos de la sala de 5 años, o quedar implícito y reponerse mediante información contextual, como se observa en el caso de la sala de 3 años -Ingrid: no no no- que condensa 'no le pongas la inyección'. Por otra parte, todos ellos tienen en común que la reiteración es utilizada como estrategia principal, es decir, la fuerza argumentativa radica en la repetición del punto de vista. También se han registrado en el corpus analizado casos en los que lo que se reitera es otra estrategia argumentativa; los hemos considerado casos de reiteración secundaria.

Asimismo, a nivel de la formulación, se observa que la reiteración puede darse en el mismo turno de habla, como ocurre en los ejemplos (6) y (7), pero también podría darse en turnos de habla diferentes, como se ha observado en otras situaciones. 


\subsubsection{Anticipación}

Las anticipaciones se constituyen como argumentos en tanto proyectan cursos de acciones deseables o no vinculados al punto de vista. Según cómo se construyan sintácticamente, se subdividen en: a) anticipación por afirmación de un hecho futuro -presenta verbos o perífrasis verbales que indiquen hechos futuros; b) anticipación de un hecho hipotético - se organiza en torno a una construcciones condicionales; c) anticipación por la afirmación de fines - presenta construcciones finales. El siguiente ejemplo fue registrado entre niños de 3 años y constituye un caso de afirmación de un hecho futuro. Un grupo de 4 niños varones han elegido jugar en el rincón de construcciones. Lautaro controla el juego y supervisa el desarrollo de la construcción. En este intercambio, Lautaro justifica el rechazo a la ficha que agregó Carlos alegando que con ella se desarmará la construcción.

(8)

Andrés: [A Lautaro, con un bloque en la mano.] ¿éste dijiste que no?

Lautaro: xxx

Andrés: [Desecha el bloque.]

Carlos: [Pone un bloque.]

Lautaro: NO:: \lo vas a desarmar a ése (pon) [8:20] [Carlos retrocede, Lautaro toma ése bloque y lo reacomoda.]

El siguiente ejemplo (9) registrado entre niños de 5 años, ocurrió en el rincón de construcciones. Armando, líder del juego, y Diego discuten cómo ubicar las piezas.

Armando.: ey:: $(\mathrm{xxx})$ diego:: cómo hacemos así/ [Intenta poner una madera sobre la construcción como si fuera techo.]

Diego: así mira\ [Acercándose para agarrar la madera.]

Armado.: no porque si pongo así se cae esto y esto [Señala con el bloque dos partes de la construcción.]

En el intercambio (9) se observa una anticipación del subtipo hecho hipotético. A nivel sintáctico, se trata de una construcción condicional y es una anticipación ya que proyecta una eventual situación futura vinculada al punto de vista. En este caso, Armando sostiene que no se debe colocar la pieza en la construcción como lo propone su compañero; pues esa acción produciría la caída de otras fichas de la construcción, hecho que asume indeseable para ambos. Es de destacar que el uso de sintagmas condicionales solo se registró entre los niños de 5 años; si bien entre los 
niños de 3 años apareció una vez, el niño que la produjo estaba jugando a dramatizar el cuento tradicional 'Los tres chanchitos' y estaba reproduciendo el texto. Entre los niños de 4 años, no se registraron casos de ese subtipo, aunque ello no constituye evidencia de que los niños de esa edad no puedan formularlos.

\subsubsection{Narración}

Los resultados del análisis mostraron el empleo por parte de los niños de una estrategia a la que denominamos 'narración'. Esta estrategia comprende aquellos casos en los que los niños refieren a hechos pasados como evidencia para sostener su posición. Hemos considerado la presencia de al menos un verbo en pretérito perfecto simple, modo indicativo, para incluir un caso particular dentro de esta categoría de estrategia. El ejemplo (10), correspondiente a una disputa entre niños de 3 años, fue registrado durante el juego de construcciones. Lautaro, Alejo, Carlos y Andrés han decidido representar el cuento 'Los tres chanchitos' con las construcciones que están desarrollando. Aunque no se han puesto de acuerdo en aspectos centrales del guión, si la construcción/casa de Lautaro es de cemento, el juego ha comenzado. Previo al siguiente intercambio, Alejo, en el rol de lobo, ya ha destruido la construcción de Carlos.

Lautaro: [Apilando bloques] TODAVÍA NO::/ LO:BO:\[20:21. Carlos y Andrés, que están a la izquierda de Lautaro, también trabajan en la reconstrucción de una casa]

Carlos: rápido rápido que $\mathrm{xxx} \backslash$

Lautaro: [Repentinamente Alejo reaparece corriendo y, con los pies, destruye la obra de Lautaro] NO::::: [ceño fruncido] [20:44]

\section{Alejo: [mirando a Carlos] te dije que venía xxx el lobo\}

En el ejemplo anterior, Alejo refiere una acción pasada para validar su accionar: como él había avisado que venía el lobo, sus compañeros no pueden cuestionar el que les haya destruido sus casas.

El ejemplo correspondiente a niños de 4 años fue registrado durante un juego de mesa del cual participaban dos pequeños, Martín y Carlos. El juego consistía en pescar figuras de peces con una espátula.

Carlos: [Acaba de volver a poner las fichas que tenía en su plato de nuevo dentro del tablero] dale ahora yo $\backslash$. primero yo $\backslash$ [Pesca algunas fichas] E::y: $\backslash$ ponelo todo A::HÍ:/ [Se refiere a las fichas que Martín tiene en su poder y que no ha vuelto a poner en el tablero] poné el otro amarillo\[Martín pone las fichas] yo ya (gané)\ 
Martín: DAme: Eso [Martín le saca la espátula a Carlos. Pesca algunas fichas]

Carlos: [Recupera la espátula]

Martín: DA::ME [Vuelve a agarrar la espátula y junta muchos peces. Ríe. Toca el pecho de Carlos] y::/ hola:: [A Carlos, quien toma la espátula y junta otros peces. Luego de dejarlos, vuelve a repetir la acción de pescar] a:: ya está::/ no VA::lel

Carlos: sí VA:le\voy a contar $\backslash$ [Cuenta sus fichas] uno dos tres cuatro cinco\ [Cuenta las fichas de Martín] uno dos tres cuatro cinco\ cinco\empate\[Martín se ríe mientras agrega más peces a acababa de contar Carlos] no porque $=$ estás haciendo- $=$ $<0.1>$ trampa $\backslash$

\section{Martín: =PERDISTE PERDISTE $=$}

\section{Carlos: trampa hiciste\}}

Martín: no:: yo: no.

En el ejemplo (11), se observan dos disputas. La primera gira en torno a los turnos del juego: Carlos pretende 'pescar' dos veces en el mismo turno, lo cual provoca el rechazo de Martín, quien solo expresa su posición sin estrategia argumentativa alguna. Carlos, por su parte, se defiende del mismo modo -Martín: no VA::le\; Carlos: sí VA:le -. Luego Carlos cuenta las fichas que cada uno recogido y declara un empate, pero Martín agrega dos fichas a su pozo, por lo que declara que Carlos ha perdido. Carlos rechaza esa resolución refiriendo las acciones pasadas de Martín como acciones que violan las reglas de juego - Carlos: trampa hiciste\.

\subsubsection{Descripción}

El análisis realizado muestra que los niños presentan ciertas características particulares de un objeto, un evento o un estado interno, que permiten afirmar el punto de vista como conclusión. Hemos denominado a esta estrategia, por tanto, descripción. La regla de traspaso que conduce a la conclusión puede quedar implícita e inclusive también puede quedar implícita la conclusión. El caso que ilustra dicha categoría para los niños de 3 años ocurrió durante un juego de dramatización en el que los niños representaban la visita al doctor. Ingrid y Juan juegan al están sentados alrededor de una mesa atendiendo a un bebote. Ambos le sujetan la cabeza mientras la niña le aplica una inyección. Luego, se acerca Ezequiel en el rol de médico.

Ezequiel: le voy a pinchar un poco [le pone una inyección al bebote]

Ingrid: NO (jugá con lu::r) LE HUELE\[Ingrid y el niño se avalanzan sobre el bebote, Ingrid le pone una inyección en la boca, Juan le toca los ojos y la frente.] 
En el intercambio anterior se puede observar cómo Ingrid recurre a la descripción de un estado interno, en este caso del bebé -NO (jugá con lu::r) LE HUELE-, como justificación para detener las acciones que Ezequiel, en rol de doctor, estaba llevando a cabo sobre el bebote. Podría pensarse que el niño invoca estratégicamente como una premisa una regla de traspaso moral, que establece que no se debe provocar dolor a otras personas.

Observemos, el ejemplo (13), registrado durante una situación de juego de construcción de la que participaron 4 varones de 5 años, Armado, Diego, Lucas y Alejandro. Han ocurrido varias disputas entre Alejandro y sus compañeros desde el inicio del juego, que han tenido como resultado su exclusión del mismo. En ese contexto ocurre el siguiente intercambio.

Alejandro: VAmos a destruir el CO::so: [Se baja del camión y lo direcciona hacia la construcción.]

Armando: e:y este quiere:. romper to:do [Mira a Alejandro con expresión de preocupación.

Alejandro amaga chocar la construcción con el camión. Armando frena el camión, comienzan a forcejear. Se escuchan silbidos de la maestra que llaman la atención de los niños, quienes disminuyen la intensidad del forcejeo. Armando se posiciona delante de Alejandro.]

Armando: [Mira a Lucas, sigue trabando el camión de Alejandro, de espaldas a Alejandro.

La maestra les silba nuevamente] che lucas alejandro no juega porque quiere romper esto [Continúa forcejeando con Alejandro].

En el intercambio entre los niños de 5 años, Armando se dirige a Lucas para justificar la exclusión de Alejandro mediante una descripción de las intenciones de su contrincante -che lucas alejand ro no juega porque quiere romper esto-. Esta estrategia deja en evidencia la acción de Alejandro, que va en contra de las reglas institucionales. Además, tiene como destinatario indirecto a la maestra, quien ya había focalizado su atención en lo que estaba ocurriendo en el rincón. Luego se inicia un nuevo forcejeo, por lo que la maestra primero llama la atención de los niños nuevamente con silbidos y luego se acerca al rincón. La presencia de la maestra hace que se disipe el conflicto. Alejandro desiste de destruir la construcción; Armando, por su parte, se ve obligado a aceptar la participación de Alejandro, porque excluir a un compañero también atenta contra las normas institucionales. 


\subsubsection{Generalización}

Esta estrategia consiste en presentar como prueba, para afirmar el punto de vista, una afirmación que conceptualiza con un cierto grado de abstracción los fenómenos que constituyen el objeto de la disputa. Un caso que ilustra esta categoría es el (14), registrado durante una situación de juego dramático entre niñas de 5 años. Rocío y Milagros han decidido ser madre e hija y atender el supermercado: Milagros es la cajera. Organizan la fruta y verdura. Luego Milagros le da plata a Rocío de la caja registradora y Rocío se va de la escena. En ese contexto aparece Karen, cuyo rol en el juego, en este segmento, no está claro.

Milagros: QUÉ:?

Karen: me das plata?

Milagros: cuánto?

Karen: COMO LA DE ROcí:o\

Milagros: [le da dinero] tomá monedas también\[le da monedas de papel]

Karen: [contando] como le diste a RoCÍ:o\

Milagros: porque R:Ocío JU::N:ta:: [4:00] y acá hay que juntar para los cliE::Ntes [Karen mira de reojo la cajá registradora, con cara de afligida]

Karen: [trata de sacar dinero de la registradora]

Milagros: pero pero hay que juntar para los clientes\[tapa la caja registradora]

Inicialmente, Milagros accede a darle dinero, pero Karen no está conforme con lo recibido, por lo que esgrime una descripción que se apoya en la regla de justicia -como le diste a RoCÍ:o \-. Milagros rechaza el reclamo mediante una descripción que se combina con una generalización. La descripción- porque R:Ocío JU::N:ta:: -que sostiene el punto de vista implícito 'no te doy más', se apoya en las acciones que Rocío realiza a partir de su rol como madre y responsable, según Milagros, del supermercado. La generalización, por su parte, se apoya en el guión que la niña tiene del supermercado: -acá hay que juntar para los cliE::Ntes-y está construida mediante la forma impersonal de obligación 'hay que juntar'.

\subsubsection{Mostración}

La estrategia de mostración se encuentra a medio camino entre una estrategia verbal y no verbal y consiste en apoyar el punto de vista a través de gestos o la realización de una serie de acciones que constituyen evidencias no expresadas verbalmente. 
Un ejemplo que ilustra esta estrategia fue registrado entre niños de 3 años. En el rincón de construcciones, 4 niños construyen un castillo y Lautaro, uno de ellos, regula la edificación. Previo a este segmento, el pequeño se ha retirado del rincón para contarle a la maestra lo que estaban haciendo con sus compañeros. Alejo interviene a distancia.

Maestra: cuando terminan me llaman/ ... un gran castillo/

Alejo: ya lo armé:: \ya lo armamos\

Maestra: se fue/. el amigo/ [Fuera de escena. Lautaro y la maestra se acercan a la construcción.]

Lautaro: no lo armamos [Se posiciona frente a la construcción y pone bloques.]

Alejo: SÍ:: ASÍ SON LOS CASTILLOS SEÑO\[Señala la construcción.] no cierto que así son los castillos/ [Continúa señalando la construcción.]

Lautaro: [Parado, mueve un bloque negando.]

Maestra: es un castillo/

Alejo: SÍ: ASÍ ES UN CASTILLO [Señala la construcción]

En el intercambio (15) Alejo defiende que ha armado un castillo, punto de vista objetado por Lautaro, produciendo una generalización -SÍ:: ASÍ SON LOS CASTILLOS SEÑO $\backslash$ - que se yuxtapone con una mostración mediante un gesto deíctico. La mostración incorpora visualmente las características de la construcción que el niño no verbaliza y le permite una rápida respuesta de defensa de su punto de vista.

En el ejemplo que sigue, (16), Martín y Lucas, de 4 años, juegan a pescar figuras de peces con una espátula. Martín invalida la jugada de Carlos a partir de una estrategia de narración en la que refiere que su compañero ha hecho trampa al pescar dos figuras simultáneamente en la misma jugada. La maestra es convocada en carácter de juez.

(16)

Martín: NO VALE\SE:ÑO:\hizo TRA:Mpa:: TIENE DO:S

Maestra: por qué tiene dos/

Martín: porque tiene $=\mathrm{dos}=$

Carlos: $=\mathrm{yo}=$ no hice trampa $\backslash$ tolo hite atí [Repite el movimiento que hizo para atrapar los dos peces con la cuchara.] 
Por su parte, Carlos, como defensa, refiere las acciones que llevó a cabo y que justifican que no hizo trampa mediante un verbo de significado general -hacerjunto con un adverbio de modo que se yuxtapone con la reactuación de las acciones involucradas en su jugada. Esta estrategia le permite simplificar la exposición de la narración completa, que le implicaría, probablemente, una mayor demanda cognitiva.

\subsubsection{Apelación a la autoridad}

Esta estrategia recupera la noción tradicional de argumentum ad verecundiam, que ha sido ampliamente estudio en el ámbito de la argumentación adulta (Perelman, 1989). El punto de vista es sostenido a partir de la autoridad de otro interlocutor que lo ha afirmado previamente. En las tres franjas etarias estudiadas se han encontrado casos en los que se remite a la autoridad de la maestra, como puede observarse en el ejemplo (17), registrado entre niños de 5 años que se encontraban jugando en el rincón de construcciones. La maestra ha introducido un set de autos nuevos, los ha presentado y ha indicado que deben compartirlos. En ese contexto tiene lugar el siguiente intercambio.

Franco: me prestás. nacho\. la moto/ [Intenta agarrar la moto que

Nacho está moviendo] me prestás la moto\NAcho/

Nacho: [Continúa moviendo la moto]

Franco: DA::le $\backslash$ me prestas nacho? DA::lem QUÉ dijo la seño\}

\section{nacho?}

El ejemplo (17) presenta la estrategia de apelación a la autoridad, encarnada en la figura de la maestra, y está formulada como un discurso referido. El ejemplo (18), por su parte, presenta un subtipo de esta estrategia no considerado por Migdalek y Arrúe (2012a): una apelación a la mayoría. El intercambio fue presentado previamente en la categoría de narración y pertenece a un grupo de niños de 5 años que se encuentran jugando en el rincón de construcciones. Diego y Alejandro mantienen una disputa en la que el primer niño ha intentado excluir al segundo del juego. Esta acción se realiza por medio de una estrategia de apelación a la mayoría, en la que Diego busca el apoyo de sus compañeros de juego, entre ellos de Armando, líder del juego. Alejandro ha utilizado la estrategia de narración como defensa, como ya hemos analizado anteriormente.

\section{Diego: Dirigiéndose a Lucas y a Alejo R.] él juega/}

Alejandro: SÍ PORQUE YO ELEGÍ EL BLOQUE [A Diego, cara de enojado, le acerca el cuerpo.] 


\subsubsection{Propuesta alternativa como subtipo de negociación}

Para llegar a un acuerdo en torno a las posiciones enfrentadas en la disputa, se han observado casos en que los niños negocian la resolución de la diferencia ofreciendo una alternativa. El primer ejemplo se produce en una disputa por un objeto. En el rincón de juegos de mesa, Fiama y Macarena, de 4 años, se encuentran jugando a un juego de secuencias y se produce una disputa por las fichas.

Fiama: señorita voy a armar este [Toma una pieza.] 1OCo:: $\backslash$ BASta. no\MA:CA: [Pelea con Iara por las fichas.]

Maestra: chicas otra vez/

Fiama:[Señalando y pataleando]

Maestra: qué necesitas/ hablÁ $<0.1>$ el del gato va acá [Coloca una pieza que tenía Fiama en la mano. La maestra se aleja.]

Fiama: maca yo tenía xxx que es el más difícil el de la muñeca [Le muestra dos fichas.] MACA/ MACA/ YO te doy este que es más difícil el de la muñeca\ mirá/ tomá/ [Le alcanza una ficha.] tomá MACA.. MACA [Cara de puchero, dándole las fichas a su compañera.]

En este intercambio, Fiama intenta recomponer la situación ofreciendo a su compañera una pieza valiosa del juego, aunque no logra su objetivo.

\subsubsection{Cortesía}

El análisis ha evidenciado que los niños utilizan diversos recursos de mitigación para lograr que su punto de vista sea aceptado. El primer ejemplo que ilustra la categoría fue registrado entre los niños de 3 años durante la dramatización de la visita al médico. Se trata de un juego del que participa toda la sala, aunque en pequeños grupos distribuidos en mesas. En el fragmento, Ingrid se encuentra jugando sola, curando a un bebote. Morena se acerca, pero pretende desplegar otro juego vinculado a escenas de la vida familiar.

Morena: la PAva/ .. LA PAVA/

Ingrid: qué/

Morena: la paval

Ingrid: acá [Amaga a darle la pava, pero luego no la suelta, forcejean. Ingrid hace que moja el algodón con la pava.] 
Morena: me DA:/

Ingrid: no:

Morena: VAmo a juga calentar el MAte/

Ingrid: NO:

Morena: VAMO a tomar MAte/

Ingrid: [Sigue jugando con la pava.]

Morena: vamo a tomar mate y: y: [Agita los brazos.] tomamos \dale/

Ingrid: no\

Morena: tomamo mate/ TOMAMO EL MATE/(xxx) [Ingrid apoya la pava y Morena la toma.]

Morena: [Toma un plato y un cuchillo que hay sobre la mesa y gira, quedando de espaldas. Se dirige a otro niño.] tomá el platito para el mate [Se aleja].

El recurso del cual se vale Morena para mitigar a imposición del punto de vista es la modalidad interrogativa. La niña le reitera la propuesta de jugar a escenas de la vida cotidiana con sutiles variaciones. Ante la negativa de Ingrid, convierte la aserción 'VAMO a tomar MAte/' en una pregunta, 'vamo a tomar mate y: y: [Agita los brazos.] tomamos $\backslash$ dale/'. De esta forma, intenta mitigar la imposición inicial que no sirvió para sumar a Ingrid al juego que ella proponía. Sin embargo, esta estrategia también fracasa y vuelve a utilizar la reiteración de la aserción, emitida con un aumento del tono de voz, nuevamente tratando de imponer su posición.

\section{Discusión y conclusiones}

Los resultados han puesto de manifiesto que en las situaciones lúdicas, cuando el contexto situacional y/o discursivo lo demanda, los niños pueden producir diversas estrategias argumentativas para sostener sus posiciones a partir de su experiencia y del conocimiento del mundo social, que incluye el institucional del jardín de infantes.

El sistema de categorías para analizar las estrategias argumentativas elaborado a partir de disputas en situaciones de juego en el contexto del hogar de distintos grupos socioculturales (Migdalek \& Arrúe, 2012a) permite dar cuenta conceptualmente de los intercambios que tienen lugar en el marco de disputas en situaciones de juego en el jardín de infantes. El sistema de categorías muestra, a diferencia de otros trabajos (Eisenberg, 1987) las estrategias argumentativas empleadas por los niños en los distintos contextos en los que se socializan, las cuales no pueden ser meramente agrupadas en una categoría gobal de soporte verbal (Eisenberg, 1987). En efecto, los resultados obtenidos en este estudio muestran, al igual que en en el análisis 
de las diputas en el contexto del hogar (Migdalek \& Arrúe, 2012a) que los niños despliegan una variedad de estrategias argumentativas verbales en la interacción. A fin de atender a las diferencias contextuales entre ambos estudios, tal como se plantea en los uno de los objetivos, el análisis realizado identificó ciertos aspectos no presentes en el marco del hogar. En particular, como también ha sido señalado por otros investigadores (Cobb-Moore, et al., 2009) las reglas institucionales propias de los espacios educativos, se destacan como elementos que deben ser considerados para comprender cabalmente el uso de las estrategias argumentativas que hacen los pequeños en situaciones de juego en el jardín de infantes. Esto puede explicar, por ejemplo, la ausencia de agresiones verbales, que sí han sido registradas en contextos hogareños (Eisenberg, 1987; Migdalek \& Arrúe, 2012a).

Asimismo, en relación con los dos primeros objetivos, la metodología empleada articuló el análisis inductivo de los datos, propio del modo de operar cualitativo (Glaser \& Strauss, 1967; Strauss \& Corbin, 1991) con el uso heurístico de categorías desarrolladas en las teorías argumentativas previas, de modo tal de describir y delimitar cada una de las categorías. En este sentido, las diversas estrategias argumentativas identificadas en el presente análisis muestran vínculos tanto con categorías que conceptualizan el argumentar adulto como con los resultados de los estudios antecedentes.

En relación con la estrategia de de reitaración ella se vincula con el recurso de la repetición estudiado en detalle desde la retórica clásica hasta la contemporánea (Perelman, 1989; Tindale, 1999). Otros estudios sobre la argumentación infantil también han identificado la reiteración como un recurso argumentativo infantil y se la ha considerado como una estrategia argumentativa simple, en tanto no aporta nueva información como argumento (Eisenberg, 1987; Dunn \& Munn, 1987; Stein \& Albro, 2001). En este sentido, se ha denominado 'estructura circular' en las investigaciones antecedentes. Sin embargo, el aporte del presente trabajo reside en mostrar la diferencia entre la repetición utilizada como estrategia principal, que coincide con los usos ya señalados, y su empleo, como estrategia argumentativa secundaria, en combinación con otra estrategia argumentativa.

Por su parte, en relación con la estrategia de anticipación, resulta pertinente señalar que algunos de sus usos coinciden con la 'argumentación pragmática' (Watson et al., 2008), esto es, aquella estructura argumentativa a través de la que el hablante recomienda -con grados distintos de fuerza y énfasis, desde la simple sugerencia hasta la amenaza- tomar o no tomar cierto curso de acción indicando las consecuencias (in) deseadas.

En relación con la estrategia de narración, ella establece vínculos con los hallazgos de Rosemberg, Silva y Stein (2011), quienes han señalado el uso de la narración como evidencia factual para sostener un punto de vista. También, Carranza (1997) ha observado el uso de la narrativa con fines argumentativos en adultos. El subtipo de 
apelación a la mayoría, identificado en este trabajo, se incluye dentro de la estrategia de apelación a la autoridad descripta por Migdalek y Arrúe (2012a). Refleja el uso de la superestructura comunicativa de la coalición como forma de poder, persuasión y toma de decisión (Walton, 2006). Finalmente, en relación con la estrategia mitigación, su uso ya había sido señalado también por Eisenberg (1987), aunque no había ahondado en los recursos lingüísticos concretos con los que se llevaba a cabo.

En relación con el objetivo d, referido a observar y analizar el uso implícito o explícito de la noción de punto de vista, hemos observado que el punto de vista puede encontrarse implícito o sintetizado y puede reconstruirse a partir de los turnos de habla precedentes o de información contextual. También se han registrado, aunque escasamente, argumentos completos con el orden conclusión-razón, coincidentemente con lo hallado por Peronard (1991).

Cabe señalarquelos argumentos esgrimidos porlos niños corresponden mayormente a 'argumentos de acción' (van Dijk, 1983; Peronard, 1991), aunque desde las teorías de la argumentación como la pragma-dialéctica no constituirían puntos de vista genuinos (van Eemeren \& Grootendorst, 2004; van Eemeren, 2010). Consideramos, sin embargo, que desde una perspectiva interpretativa de la investigación científica como la adoptada en el presente trabajo, que subraya la comprensión del fenómeno en estudio y el carácter contextualizado del análisis (Gibaja, 1986; Forni, 1993; Sirvent, 2003) no cabe rechazar la naturaleza argumentativa de los mismos.

Lo importante a rescatar es que el niño manifiesta lingüísticamente un conflicto cognitivo con cierta intensidad (Desalles, 2007, 2011), requerimiento necesario, pero no suficiente, para desencadenar argumentación, en tanto proceso social (Walton, 2006), y crear el constructo argumento, en tanto producto de un individuo. El conflicto cognitivo es necesario pero no suficiente para que el hablante adopte lo que Desalles (2007) llama el 'modo argumentativo', esto es, aquel estado en que un agente intenta encontrar una solución a un conflicto cognitivo -en este caso la presión del grupo para que siga jugando, y el deseo del niño de parar de jugar-. Según Desalles (2007) cuando los humanos estamos en el modo argumentativo detectamos y resolvemos conflictos cognitivos; éstos aparecen cuando las creencias y los deseos que constituyen el conflicto tienen un grado importante de intensidad contradictoria. Dicho de otra forma, tenemos conflictos cognitivos cuando tenemos representaciones diferentes de una situación dada o respecto de los términos a través de los que definimos o enmarcamos la situación.

Podríamos coincidir con otros autores (Desalles, 2007; Mercier, 2011; Mercier \& Sperber, 2011) en que la necesidad de sortear estratégicamente desafíos a las creencias, deseos y otros estados intencionales de los niños en contextos de comunicación lingüística, es el impulso al desarrollo de la subcompetencia argumentativa. El comportamiento protoargumentativo estratégico es, desde el punto de vista del desarrollo de la facultad cognitiva, el inicio del robustecimiento de argumentar que 
alcanza su madurez con la exposición lingüística explícita de razones produciendo inferencias consistentes, válidas y sólidas, atendiendo a parámetros pragmáticos como la suficiencia, la relevancia y la aceptabilidad (Johnson, 2000).

En relación con el objetivo c, en las distintas franjas etarias analizadas hemos registrado intercambios en los que se enfrentan puntos de vista sin un despliegue argumentativo que los sostenga. Esta expresión de oposición muestra claramente que el niño mantiene una posición distinta de la de su oyente que podría provocar, eventualmente, la expresión de argumentos (razones) específicos que justifiquen tal posición. Es por ello que la expresión de oposición puede considerarse como la condición de posibilidad para el desarrollo de la competencia argumentativa. Futuras investigaciones deberían considerar qué factores influyen para que tenga lugar tal despliegue argumentativo; en el contexto institucional se deberá indagar especialmente al rol que la maestra puede ocupar en el desencademiento de un argumento por parte de los niños.

Asimismo, en relación con las diferencias etarias en el empleo de las estrategias, si bien todas las categorías han sido registradas para todos los rangos de edad considerados, existen diferencias en las cantidades observadas que tendrán que ser examinadas en trabajos estadísticos posteriores. De modo general, creemos que la presencia de la apelación a la mayoría, registrada en intercambios correspondientes a niños de 4 y 5 puede vincularse con las características de juego grupal, que se inicia a los 4 años y ya está consolidado a la edad de 5 años (Elkonin, 1980).

Finalmente, a partir de los resultados obtenidos por Migdalek y Arrúe (2012b), quienes identificaron diferencias significativas en el uso de ciertas estrategias argumentativas infantiles durante disputas entre pares en situaciones de juego en el hogar según el grupo social de procedencia de los niños, futuras investigaciones deberán indagar si en el contexto del jardín de infantes se registran también diferencias de la misma índole. 


\section{REFERENCIAS BIBLIOGRÁFICAS}

Bernard, S., Mercier, H. \& Clément, F. (2012). The power of well-connected arguments: Early sensitivity to the connective because. Journal of Experimental Child Psychology, 111, 128-135.

Bruner, J. (1986). El habla del niño. Barcelona: Paidós.

Carranza, I. (1997). Argumentar narrando. México: Universidad Autónoma de México.

Clément, F. (2010). To trust or not to trust? Children's social epistemology. Review of Philosophy and Psychology, 1(4), 531-549.

Cobb-Moore, C., Danby, S. \& Farrell, A. (2009). Young children as rule markers. Journal of Pragmatics, 41, 1477-1492.

Cole, M. (1999). Psicología cultural. Madrid. Morata.

Crespo, N. (1995). El desarrollo ontogenético del argumento. Revista Signos. Estudios de Lengua y Literatura, XVIII(37), 69-82.

Del Río, P. \& Álvarez, A. (2001). From activity to directivity. The question of involvement in education. En G. Well \& G. Claxton (Eds.), Learning for life in the 21st Century: Sociocultural perspectives on the future of education (pp. 59-72). Oxford: Blackwell.

Dessalles, J. L. (2007). Why we talk. The evolutionary origins of language. Nueva York: Oxford University Press.

Dessalles, J. L. (2011). Reasoning as a lie detection device. Behavioral and Brain Sciences, 34(2), 76-77.

Dunn, J. \& Munn, P. (1986). Sibling quarrels and maternal intervention: Individual differences in understanding and aggression. Journal of Child Psychology and Psychiatry and Allied Disciplines, 27(5), 583-595.

Eisenberg, A. (1987). Learning to argue with parents and peers. Argumentation, 1(2), 113-125.

Elkonin, D. (1980). Psicología del juego. Madrid: Visor.

Evans, J. (2010). Thinking twice. Two minds in one brain. Nueva York: Oxford University Press.

Faigenbaum, G. (2012). El desarrollo de las habilidades argumentativas. En J. A. Castorina \& M. Carretero (Comps.), Desarrollo Cognitivo y Educación [I]. Los inicios del conocimiento (pp. 292-313). Buenos Aires: Paidós. 
Forni, F. (1993). Estrategias de recolección y estrategias de análisis en la investigación social. En F. Forni, M. Gallard \& I. Vasilachis de Gialdino (Eds.), Métodos Cualitativos II. La práctica de la investigación (pp. 9-105). Buenos Aires: Centro Editor de América Latina.

Gibaja, R. (1986). El mundo simbólico de la escuela. Oaxaca: Universidad Autónoma Benito Juarez.

Glaser, B. \& Strauss, A. (1967). The discovery of grounded theory. Chicago: Aldine Publishing Company.

Gumperz, J. (1982). The linguistic bases of communicative competence. En D. Tannen (Ed.), Analyzing discourse: Text and talk (pp. 323-334). Washington: Georgetown University Press.

Gumperz, J. (1984). Communicative competence revisited. En D. Schiffrin (Ed.), Meaning, form and use in context: Linguistic applications (pp. 278-289). Washington: Georgetown University Press.

Johnson, R. (2000). Manifest rationality. A Pragmatic Theory of Argument. Mahwah, NJ: Lawrence Erlbaum.

Kovács, A., Téglás, E. \& Endres, A. (2010). The social sense: Susceptibility to others' beliefs in human infants. Science, 330, 1830-1834.

Leontiev, A. (1981). The problem of activity in psychology. En J. V. Wertsch (Ed.), The concept of activity in soviet psychology (pp. 37-71). Nueva York: Shape.

Luria, A. (1995). Conciencia y lenguaje. Madrid: Visor.

Mercier, H. (2011). Reasoning serves argumentation in children. Cognitive Development, 26(3), 177-191.

Mercier, H. \& Sperber, D. (2011). Why do human reason? Arguments for an argumentative theory. Behavioral and Brain Sciences, 34(2), 57-74.

Migdalek, M. \& Arrúe, J. (2012a). Habilidades argumentativas de niños de cuatro años: Un análisis de las disputas en las situaciones de juego en hogares de niños de población urbano marginada y sectores medios. Ponencia presentada en las Terceras Jornadas Internacionales de Investigación y Prácticas en Didáctica de las lenguas y las literaturas, Bariloche.

Migdalek, M. \& Arrúe, J. (2012b). Habilidades argumentativas en niños de 4 años en el marco de disputas durante el juego en el hogar: diferencias entre niños de sectores sociales medios y niños que viven en poblaciones urbano marginadas. Ponencia presentada en el IV Congreso Internacional de Investigación y Práctica Profesional en Psicología, XIX Jornadas de Investigación y VIII Encuentro de Investigadores del MERCOSUR, Buenos Aires. 
Mondada, L. \& Pekarek, S. (2001). Interactions acquisitionnelles en contexte: Perspectives théoriques et enjeux didactiques. Le Français Dans le Monde, Numeró Spécial: Théories linguistiques etenseignement du français aux non-francophones (juillet), 107-142.

Nelson, K. (1996). Language in cognitive development. Nueva York: Cambridge University Press.

Pekarek, S. (2006). Compétence et langage en action. Bulletin Suisse de Linguistique Appliquée, 84, 9-45.

Perelman, Ch. \& Olbrechts Tyteca, L. (1989). Tratado de la argumentación. La nueva retórica. Madrid: Gredos.

Peronard, M. (1991). Antecedentes ontogenéticos de la argumentación. En E. Luna Traill (Coord.), Scripta philologica: In honorem Juan M. Lope Blanch (pp. 417-443). México: Universidad Autónoma de México.

Rosemberg, C. (2008). El lenguaje y el juego en la educación infantil. En P. Sarlé (Coord.), Enseñar en clave de juego: Enlazando juegos y contenidos (pp. 61-75). Buenos Aires: Novedades Educativas.

Rosemberg, C., Silva, M. \& Stein, A. (2011). Narrativas infantiles en contexto: Un estudio en hogares de barrios urbano marginados de Buenos Aires. Revista de Instituto de Investigaciones en Ciencias de la Educación, 28, 1-21.

Seidman, S., Nelson, K.\& Gruendel,J. (1986). Make believe scripts: The transformation of ERs in fantasy. En K. Nelson (Ed.), Event knowledge: Structure and function in development (pp. 161-187). Hillsdale, NJ: Lawrence Erlbaum.

Silvestri, A. (2001). La producción de la argumentación razonada en el adolescente: Las falacias de aprendizaje. En M. C. Martínez (Ed.), Aprendizaje de la argumentación razonada. Desarrollo temático de los textos expositivos y argumentativos (pp. 29-48). Cali: Cátedra UNESCO para la Lectura y la Escritura, Universidad del Valle.

Sirvent, M. (2003). El proceso de investigación. Buenos Aires: Editorial Universidad de Buenos Aires.

Stanovich, K. (2011). Rationality and reflective mind. Nueva York: Oxford University Press.

Stein, N. \& Albro, E. (2001). The origins and nature of arguments: Studies in conflict understanding, emotion, and negotiation. Discourse Processes, 32(2-3), 113-133.

Strauss, A. \& Corbin, J. (1991). Basics of qualitative research. Nueva York: Sage.

Surian, L., Caldi, S. \& Sperber, D. (2007). Attribution of beliefs by 13-month-old infants. Psychological Science, 18(7), 580-6. 
Tindale, C. (1999). Acts of arguing. A rhetorical model of argument. Albany, Nueva York: State University of New York Press.

Tindale, C. (2004). Rhetorical argumentation. Nueva York: Sage.

Tomasello, M. (2008). Origins of human communication. Cambridge, Mass.: The MIT Press.

Tomasello, M. (2010). Why we cooperate. Cambridge, Mass.: The MIT Press.

Tusón, A. (1995). Análisis de la conversación. Barcelona: Ariel.

Van Dijk, T. (1983). La ciencia del texto: Un enfoque interdisciplinario. Barcelona: Paidós.

Van Eemeren, F. (2010). Strategic maneuvering in argumentative discourse. Extending the pragma-dialectical Theory of Argumentation. Amsterdam: John Benjamins.

Van Eemeren, F. \& Grootendorst, R. (2004). A systematic Theory of Argumentation. The pragma-dialectical approach. Nueva York: Cambridge University Press.

Vygotski, L. (1964). Pensamiento y lenguaje. Buenos Aires: Editorial Lautaro.

Walton, D. (2006). Fundamentals of critical argumentation. Nueva York: Cambridge University Press.

Walton, D., Reed, C. \& Macagno, F. (2008). Argumentation schemes. Nueva York: Cambridge University Press.

Wertsch, J. (1998). La mente en acción. Buenos Aires: Aique.

Wyman, E., Rakoczy, H. \& Tomasello, M. (2009). Normativity and context in young children's pretend play. Cognitive Development, 24, 146-155.

Zadunaisky Ehrlich, S. \& Blum-Kulka, S. (2010). Peer talk as a 'double opportunity space': The case of argumentative discourse. Discourse Society, 21(2), 211-233. 


\title{
NOTAS
}

1 Se trata de "La regulación de las situaciones de juego en la educación infantil" enmarcada en el programa UBACYT “El lenguaje, el juego y la Educación Infantil”, Facultad de Filosofía y Letras, Universidad de Buenos Aires.

2 Las situaciones analizadas en este trabajo fueron recogidas en el marco del "Programa Oscarcito. Desarrollo Lingüístico y Cognitivo en la infancia" dirigido por la Dra. Celia Renata Rosemberg y Ana María Borzone, que cuenta con el apoyo del CONICET- CIIPME, la Fundación Care de Alemania y la Fundación Arcor de Argentina.

3 Dichas poblaciones están conformadas por viviendas precarias, mayormente construidas con madera y materiales recuperados y los servicios de infraestructura son insuficientes o inexistentes. Aunque la mayoría de estos barrios tiene conexiones de agua potable, todos ellos carecen de cloacas y gas natural. En muchos casos se provee de electricidad mediante conexiones clandestinas a la red. El acceso al barrio es por pasillos estrechos de tierra o de cemento con piso. En algunas de estas poblaciones, la lluvia produce el anegamiento de los accesos, por lo que los días de lluvias fuertes los niños no pueden concurrir al jardín de infantes. En la Ciudad de Buenos Aires (Argentina), 116.000 personas viven en 'villas de emergencia', y en las afueras de la ciudad, otras 1.114.500 personas viven en estas condiciones (fuente: Estadísticas del Gobierno de la Ciudad de Buenos Aires, Argentina).

\section{ANEXO}

\section{Glosario de símbolos de transcripción utilizados}

\author{
PALABRA MAYÚSCULA énfasis \\ $==$ superposición \\ . pausa menor a 0.1 . segundos \\ $(\mathrm{xxx})$ inaudible \\ (dudoso) fragmento dudoso \\ [] fenómenos no léxicos \\ :: alargamiento vocálico y consonántico \\ / tono ascendente \\ $\backslash$ tono descendente
}

